\title{
Simulation in Angiography - Experiences from 5 Years Teaching, Training, and Research
}

\section{Simulation in der Angiografie - Erfahrungen aus 5 Jahren Lehre, Ausbildung und Forschung}

Authors

Kornelia Kreiser, Kim Gehling, Claus Zimmer

Affiliation

Department of Diagnostic and Interventional Neuroradiology, Klinikum rechts der Isar, Technical University Munich,

München, Germany

Key words

cerebral angiography, interventional procedures, education, simulation

received 25.05.2018

accepted 03.10.2018

Bibliography

DOI https://doi.org/10.1055/a-0759-2248

Published online: 12.2.2019

Fortschr Röntgenstr 2019; 191: 547-552

(c) Georg Thieme Verlag KG, Stuttgart · New York

ISSN 1438-9029

Correspondence

Frau Dr. Kornelia Kreiser

Neuroradiologie, Klinikum rechts der Isar der Technischen

Universität München, Ismaninger Str. 22, 81675 München,

Germany

Tel.: ++ 49/89/41404651

kornelia.kreiser@tum.de

\section{ABSTRACT}

Purpose The example of university radiology/neuroradiology illustrates how high-tech angiography simulators can be used meaningfully in teaching, clinical training and research.

Materials and Methods/Technical Basics A VIST LAB simulator (Mentice, Gothenburg, Sweden), which has been continuously developed both in terms of software and hardware, has been in use since 2013. Recently, the simulator has been integrated into the angiography suite Azurion (Philips, Amsterdam, Netherlands).

Results/Areas of Application In student education there is the possibility for intensive examination of cerebrovascular diseases and their therapy in small group lessons. The training of beginners in diagnostic and interventional angiography begins mandatorily on the simulator. Research questions are the proof of validity and the training effect, but also the influence on patient safety and the possible cost reduction of an intervention.
Conclusion As a result of continuous further development in recent years, simulators are now very well suited for both student teaching and beginner medical training. In the future, even experienced interventionalists could benefit from further technical advances, which should also be driven by academic research. Possible effects would be the reduction of examination times, complications and costs.

Key Points:

- Angiography simulators are useful in teaching students, medical training and research.

- Linking a simulator to an angiography suite increases the degree of reality even further.

- Real patient cases can be practiced and thus patient safety can be increased.

- Future developments should also increase the benefit for experienced interventionalists.

- Integration of simulators into certification programs (e. g. DEGIR) is to be targeted in the future.

\section{Citation Format}

- Kreiser K, Gehling K, Zimmer C. Simulation in Angiography Experiences from 5 Years Teaching, Training, and Research. Fortschr Röntgenstr 2019; 191: 547-552

\section{ZUSAMMENFASSUNG}

Ziel Am Beispiel einer universitären Radiologie/Neuroradiologie wird verdeutlicht, wie Hightech-Angiografiesimulatoren in Lehre, klinischer Ausbildung und Forschung sinnvoll eingesetzt werden können.

Material und Methoden/Technische Grundlagen Seit 2013 ist ein VIST LAB-Simulator der Firma Mentice (Göteborg, Schweden) im Einsatz, der bis heute sowohl hinsichtlich Software als auch Hardware kontinuierlich weiterentwickelt wurde. Seit Kurzem ist der Simulator in die Angiografieanlage Azurion (Philips, Amsterdam, Niederlande) integriert.

Ergebnisse/Einsatzbereiche In der studentischen Ausbildung besteht in fakultativem Kleingruppenunterricht die Möglichkeit für eine intensive Auseinandersetzung mit zerebrovaskulären Krankheitsbildern und deren Therapie. In der ärztlichen Weiterbildung beginnt die Ausbildung von Anfängern in diagnostischer und interventioneller Angiografie obligatorisch am Simulator. Forschungsfragen sind der Nachweis der Validität und des 
Trainingseffekts, aber auch der Einfluss auf die Patientensicherheit und die mögliche Kostenreduktion einer Intervention.

Schlussfolgerung Als Resultat kontinuierlicher Weiterentwicklung der letzten Jahre sind Simulatoren mittlerweile sowohl im Studentenunterricht als auch in der ärztlichen Anfängerausbildung sehr gut einsetzbar. Von weiteren techni- schen Fortschritten, die auch durch akademische Forschung vorangetrieben werden sollten, könnten in Zukunft auch erfahrene Interventionalisten profitieren. Mögliche Effekte wären die Reduktion von Untersuchungszeiten, Komplikationen und Kosten.

\section{Introduction}

Simulators have long been a mandatory part of practice and competence testing in aviation [1]. Although high-tech simulation has only become more widely used in the medical field in recent years, it is gaining increasing acceptance [2].

There are a number of reasons to use an angiography simulator, particularly in interventional neuroradiology:

- Since an increasing number of questions regarding cerebrovascular pathologies can be answered using noninvasive methods such as computed tomography and MRI [3 - 5], the importance of diagnostic catheter angiography has decreased in recent years. However, interventions still require expertise in the often technically challenging probing of the supra-aortic branches.

- The need for interventional neuroradiologists is steadily growing particularly in association with the continually increasing number of endovascular stroke treatments [6]. There is a need for standardized and structured training throughout Germany.

- After completing medical school, many residents start their training directly in neuroradiology where they gain their first angiography experience in the supra-aortic arteries in which every complication can have severe neurological effects. Simulators allow unlimited training possibilities, particularly in beginner training, without posing any risk to patients.

In addition to the stated reasons for using simulators in medical training, there are also numerous ways to use simulators in student teaching and research as performed at the University Hospital rechts der Isar in the last 5 years and discussed in the following.

\section{Technical background}

The simulator was purchased at the end of 2012 after the products of three manufacturers (CATHI [Mannheim, Germany], Simbionix [Cleveland, OH, USA] and Mentice [Göteborg, Sweden]) were first introduced and tested. VIST LAB from Mentice was selected since it offered biplane imaging as well as the option for the user to import individual patient datasets. The model initially represented a standalone solution. The optional portable simulator components were integrated in an adjustable-height table ( $\triangleright$ Fig. 1). In total, four monitors were used for operation of the simulator, biplane viewing of the current images, and processing of the stored series. To ensure the best possible integration in the clinical routine, a separate room in close proximity to the department to be used exclusively for simulation was provided.
The available software modules initially included "carotid stenting" and "aneurysm coiling". An update in 2015 added radiation safety software to both modules to provide feedback regarding radiation dose both for the patient and the examiner. The latest module "endovascular stroke treatment", which our department helped to develop, has been available since 2016.

The above-mentioned option of importing individual patient datasets is made possible by software integrated in the simulator called "Case-it". DICOM datasets from CT or MRI angiography examinations are segmented semi-automatically with the help of the visualization platform IntelliSpace Portal (Philips, Amsterdam, the Netherlands) ( $\triangleright$ Fig. 2a) and imported in STL (stereolithography) format into the simulator ( $\mathbf{F i g}$. $\mathbf{2 b}$ ). If the data is only available from the level of the aortic arch in an intracranial direction, selections can be made from various templates ( $\nabla$ Fig. $2 \mathbf{c}$ ) and linked with the patient anatomy ( $\bullet$ Fig. $2 \mathbf{d}$ ) to complete the anatomy from the common femoral artery to the descending thoracic aorta. Depending on the quality of the dataset, the entire process takes approximately 15 - 30 minutes per patient.

The parameters recorded and stored by the simulator include examination time, fluoroscopy time, total time for the series, number of series and amount of contrast agent. The system also records which materials were used at which time.

Even though the handling of diagnostic catheters and wires was already quite realistic and has been improved with every software update, limitations had to be accepted in other areas for a long time:

- The control panel and pedals differed from those of the angiography system in many ways.

- No C-arms were physically present and their position and angle were only visible in a small pictogram on the edge of the monitor.

- The injection of contrast agent is simulated by the injection of air. Continuous flush of the catheters is not provided or possible.

However, the purchase of a new angiography system in our department allowed extensive improvements including an even more realistic work environment. Therefore, the RFP for the system included previously unavailable integration of the simulator in the real work environment.

As a result of the successful cooperation between Mentice and Philips, simulators have been able to be used for the first time globally in a real biplane angiography suite since the start of 2018 ( $\triangleright$ Fig. 3). To do this, the simulator is placed on the angiography table and connected with the laptop situated at the foot of 


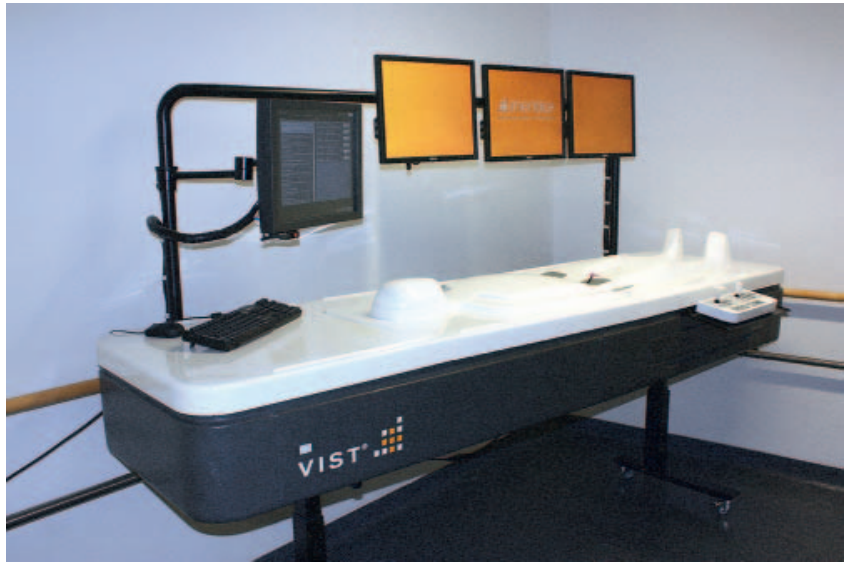

- Fig. 1 Stationary simulator (VIST LAB).
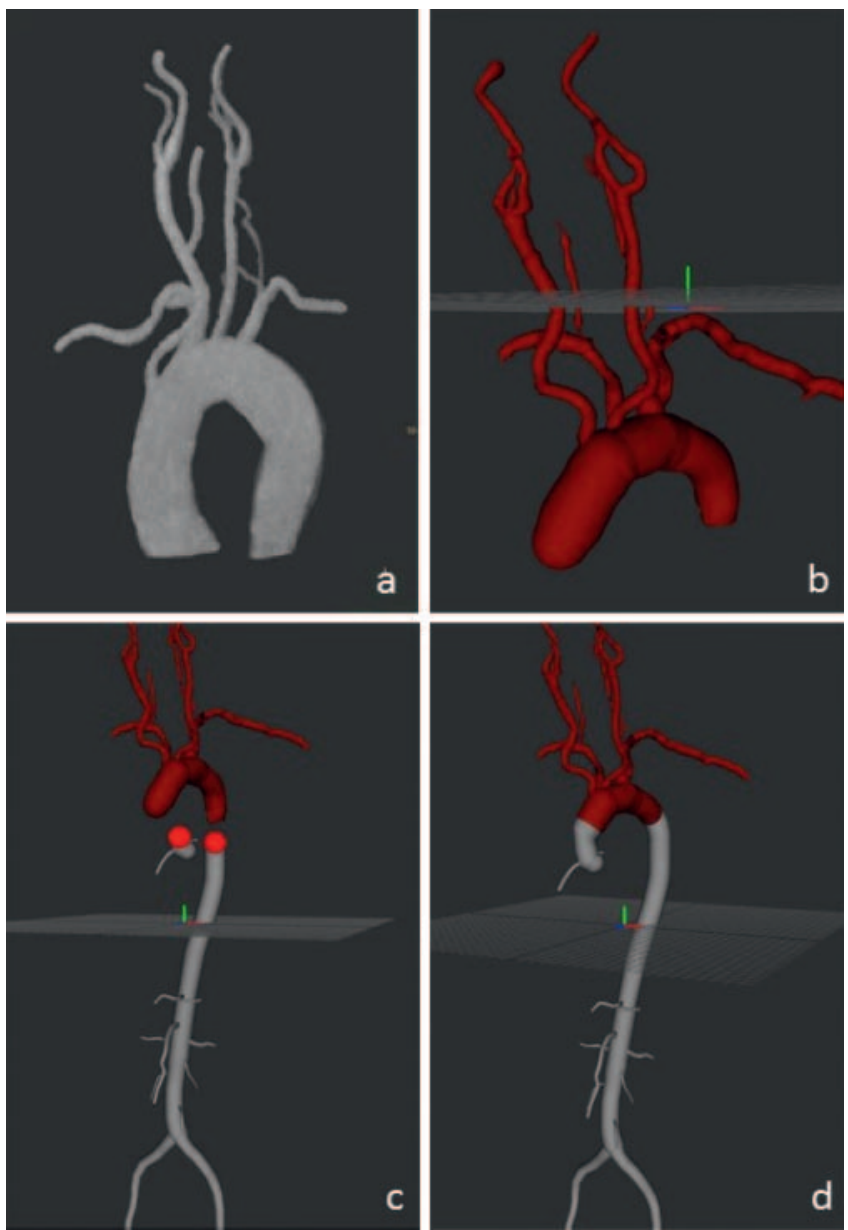

- Fig. 2 Transmission of real patients data to the simulator. a After semiautomatic segmentation. b After transmission of the STL-file to the simulator. $\mathbf{c}$ Before linking to an aortic template. $\mathbf{d}$ Complete anatomy after linking.

the table. An additional three cable connections transfer monitor and data signals from the angiography system to the simulator.

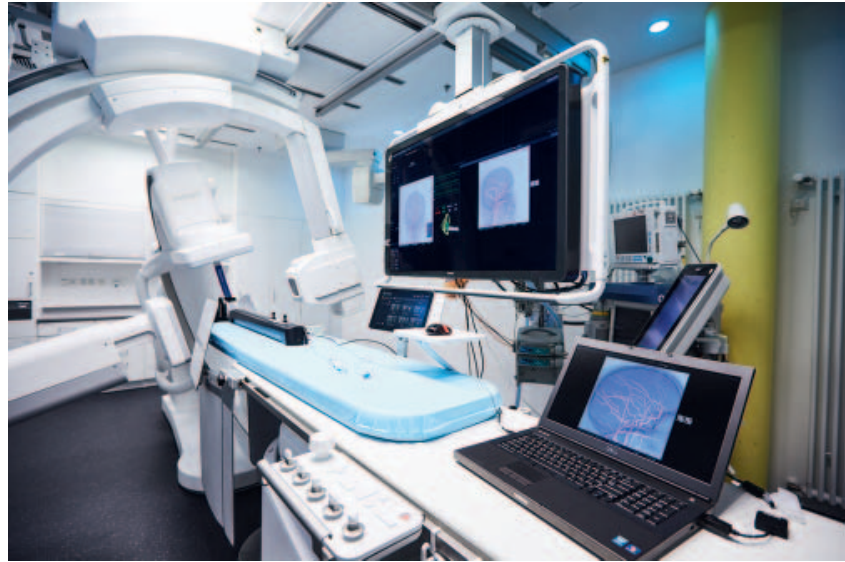

- Fig. 3 Integration of the simulator in an angiography suite.

The table position in all room planes and the position and angle of the $\mathrm{C}$-arms are detected by the simulator and can be controlled with the real control elements. The biplane image is tracked on the flatscreen of the angiography system. It takes only a couple of minutes to set up and put away the simulator so that even short breaks in the schedule can be used for training purposes.

The integration of the simulator in the angiography suite has made the simulation of angiography interventions realistic enough to be extremely beneficial, particularly for beginners, in our experience. The ability to learn all system-related processes in advance allows residents to concentrate more fully on other challenges when performing the first real angiography examination: A real patient who is capable of moving requires communication, and manipulation in perfused vessels requires careful use of continuous flush and the prevention of air embolisms and thrombi. Moreover, the immediate evaluation of possible pathologies represents a further challenge.

The communication aspects have already been implemented by Mentice and Laerdal (Stavanger, Norway) with the integration of the simulator in an anesthesia mannequin $[7,8]$ which will be interesting particularly for team training. The use of real fluids has also been implemented in a pilot project and will be available in the coming year according to the manufacturer.

\section{Areas of application}

\section{Student education}

In the mandatory medical school curriculum, neuroradiology is integrated in the radiology curriculum or participates in interdisciplinary events with other clinical subjects. The focus is usually on diagnostic imaging in the particular subject. Even though we have offered an additional intensive required elective "neuroradiology" for a long time, the interventional aspects of the subject can only be addressed on a theoretical basis. Use of the simulator made it possible to teach students the fundamentals of performing angiography examinations and the first steps in interventions like caro- 
tid stenting, aneurysm coiling and since 2016 also endovascular stroke treatment in a new required elective, designed as a 2-day block course. To achieve the highest possible degree of active participation by the individual students, the number of participants is limited to 8 students, who are trained in parallel in two groups on the simulator and a silicone model. The fact that the course is consistently full a few minutes after the start of registration is an indication of the attractiveness of the offer even though the course content far exceeds the curricular requirements. The goal of the course is not to give students a higher level of competence in the independent performing of interventions. Rather the intensive examination of the various clinical pictures and treatment options as well as the gaining of experience with fine-motor challenges is intended to spark enthusiasm for interventional radiology/neuroradiology. Many later applicants for resident positions cite this course as a reason for being interested in our field.

\section{Residency training (diagnostic)}

Since 2013, training on the simulator has been a mandatory part of the curriculum for residents.

The first two years of training are dedicated to cross-sectional imaging and CT-guided intervention, while the third year of training includes ultrasound imaging and angiography.

Parallel to assisting in interventions and learning groin puncture, every resident must perform at least 20 diagnostic panangiography examinations on the simulator before being allowed to examine a real patient. Simulator-based training is structured as follows:

- The trainer first demonstrates a complete procedure on the simulator to show the basic principles of catheter guidance and the usual examination standards (including image settings, required planes, and series duration).

- The operation of the simulator and the selection of the materials (catheter and wires) are explained and are performed together with the resident for the first three simulations under constant supervision.

- Additional procedures can then be performed independently with at least every fifth procedure being performed under supervision in order to detect catheter behavior specific to the simulator and to point out differences with respect to reality.

- The degree of difficulty of the cases continuously increases according to the anatomical configuration of the aortic arch so that the use of sidewinder catheters is necessary to reach all supra-aortic vessels by the end of the 20 procedures.

Even after completion of the first independent real angiography examinations, it proved useful to return to the simulator again and again. In particular, the turning maneuver when using a sidewinder catheter usually presents a major challenge for beginners. The ability to practice and discuss this technique in detail on the simulator highlights one of the advantages of this training method. It, of course, is not calming to patients when they are privy to extensive discussions during an examination indicating the examiner's low level of training.

The extent to which a positive effect of a simulator on the training of residents can actually be verified and proven is the subject of scientific studies currently being performed at our institute. In detail, we are examining the extent to which the total

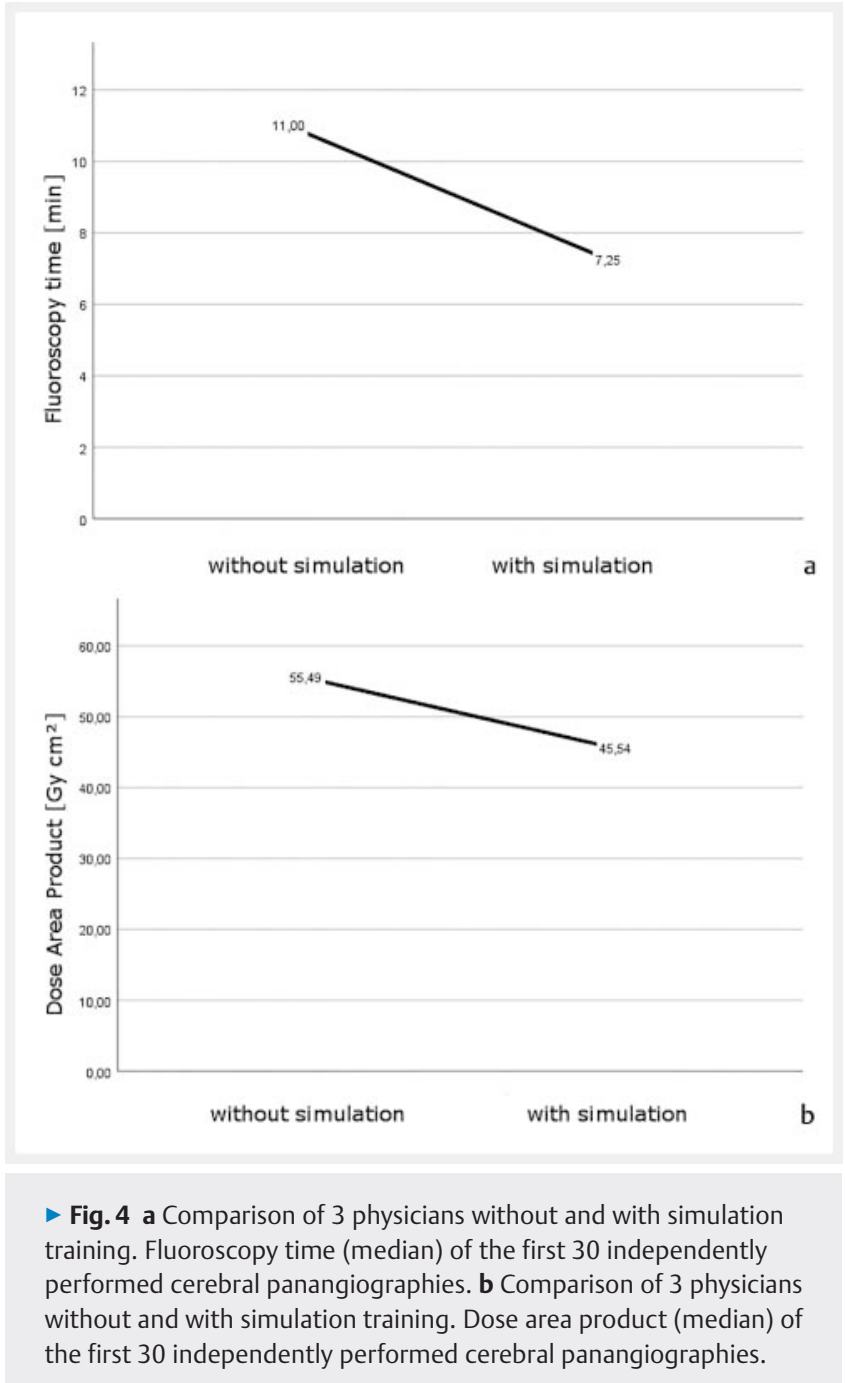

radiation dose, the amount of contrast agent, and the number of series in real examinations can be reduced by having performed previous simulations. Moreover, the influence of a previous simulation on material selection or the number of catheter changes is being examined. Hand-eye coordination as well as other parameters are being analyzed by evaluating the recordings of an eye tracking camera. Moreover, the validity of simulations, i. e., whether experts perform better than beginners in simulations with respect to the parameters above, is being examined. Finally, the training effects are of interest. We are examining whether the indicated parameters can be improved as well as whether the subjective experience and the situational stress level change after 10 simulated angiography examinations.

According to initial analyses, the benefit of this training concept can be determined by comparing residents who learned how to perform angiography prior to 2013 in the conventional manner with those who learned after 2013 based on the above-described concept. A comparison of the first 30 independently performed panangiography examinations shows a significant reduction in the radiation dose from 55.49 to $45.54 \mathrm{~Gy}^{*} \mathrm{~cm}^{2}$ and in the total fluoroscopy time from 100 to 7.25 minutes on average ( $\triangleright$ Fig. 4). 
Although only one small group of 3 physicians has been compared to date, a tendency towards effects of the modified training concept can be identified. These analyses are a work in progress.

The performance of real endovascular interventions is currently not included in our curriculum for residents and is reserved exclusively for specialists. To increase understanding of methods as well as to show the attractiveness of our field at a relatively early point in training, every resident should also perform individual supervised stroke treatment and aneurysm coiling.

\section{Residency training (intervention)}

The corresponding requirements are discussed here based on the specialists' level of training.

- Specialists without cerebral angiography experience initially follow the steps described above.

- Specialists with cerebral angiography experience not acquired in our department must perform multiple angiography examinations of varying degrees of difficulty. It is often necessary to repeatedly use the sidewinder catheter here.

- Based on the 6 cases of the stroke module, the different mechanical thrombectomy options are practiced under supervision.

However, since the haptic feedback of the materials used for interventions, like stent retrievers and coils, is not yet very realistic, the benefit of the simulator for training interventionalists is primarily the opportunity to discuss every step of the treatment in detail and the ability to repeat them as many times as desired or with the help of various techniques. Particularly in the treatment of strokes, beginners often find it difficult to identify and understand the fast sequence of individual hand movements and the variety of materials. In addition, complications are also integrated in individual simulation scenarios. After the detection and correct interpretation of complications (e.g., whether a vascular occlusion persists after a stent retriever maneuver due to thrombus or a vascular spasm), possible solutions can be tested on the simulator and discussed in detail.

\section{Research}

The validity of the use of angiography simulators has already been confirmed not only for coronary angiography examinations [9] and peripheral interventions in the iliac arteries [10] but also with respect to cerebral angiography examinations [11, 12]. In addition to the verification of training effects, research is focused on the implementation of patient-specific simulations in the clinical routine. Research is also being performed to verify the reduction of examination times also for interventions and consequently the reduction of complication rates and costs.

\section{Future and Conclusion}

In a university radiology/neuroradiology department, angiography simulators can be effectively used not only for the training of angiography beginners but also for student education and research. Manufacturers of both simulators and angiography systems need to make the training even more realistic, thereby promoting accep- tance also in non-university settings. Compatibility between simulators and angiography systems and all available functions should be provided. However, to achieve added value also for experienced interventionalists, the behavior of interventional materials, such as microcatheters, stent retrievers, and coils, must be made more realistic. In addition, real patient data should be able to be implemented even faster and with greater detail. The goal should be the realistic simulation of elective interventions, like aneurysm coiling, flow diverter implantation and embolization of an arteriovenous malformation, and the associated testing of certain materials (coils, catheters, etc.) for suitability for the particular application. This can reduce intervention time - and thus the probability of complications - and facilitate the selection of materials, so that the more targeted use of products helps to reduce costs. Simulators can be used in the development of new materials. Mandatory integration in certification programs of the professional societies (e.g. DEGIR) is also conceivable. Finally, medical competence could be verified also in a medicolegal sense by attending courses using a simulator among other things.

\section{CLINICAL RELEVANCE}

- Given the decreasing number of diagnostic catheter angiography examinations, angiography simulators offer an ideal solution for beginner training.

- The inclusion of a simulator in the environment of a real angiography suite offers the best preparation for the first examination of a patient.

- Real patient anatomy can be practiced in advance and patient safety can be increased by reducing examination times.

\section{Conflict of Interest}

The authors declare that they have no conflict of interest.

\section{References}

[1] Rolfe JM, Staples KJE (eds.) Flight simulation. Cambridge Aerospace series; Cambridge University press; 1988

[2] Bradley P. The history of simulation in medical education and possible future directions. Med Educ 2006; 40: 254-262

[3] Jäger HR, Grieve JP. Advances in non-invasive imaging of intracranial vascular disease. Ann R Coll Surg Engl 2000; 82: 1 -5

[4] Lu L, Zhang L], Poon CS et al. Digital subtraction CT angiography for detection of intracranial aneurysms: comparison with three-dimensional digital subtraction angiography. Radiology 2012; 262: 605-612

[5] Pjontek R, Önenköprülü B, Scholz B et al. Metal artifact reduction for flat panel detector intravenous $C T$ angiography in patients with intracranial metallic implants after endovascular and surgical treatment. J Neurointerv Surg 2016; 8: 824-829

[6] Berlis A, Weber W. Nationwide care for acute ischemic stroke patients is ensured by radiologists and neuroradiologists. Rofo 2017; 189: 303-308

[7] www.mentice.com/laerdal-simman-vascular [Internet, cited 2018 Jun 24]

[8] www.laerdal.com/de/products/simulation-training/emergency-caretrauma/simman-vascular [Internet, cited 2018 Jun 24] 
[9] Jensen UJ, Jensen J, Olivecrona GK et al. Technical skills assessment in a coronary angiography simulator for construct validation. Simul Healthc 2013; 8: 324-328

[10] Bech B, Lönn L, Falkenberg $M$ et al. Construct validity and reliability of structured assessment of endovascular expertise in a simulated setting. Eur J Vasc Endovasc Surg 2011; 42: 539 - 548
[11] Spiotta AM, Rasmussen PA, Masaryk TJR et al. Simulated diagnostic cerebral angiography in neurosurgical training: a pilot program. J Neurointerv Surg 2013; 5: 376-381

[12] Nguyen N, Eagleson R, Boulton M et al. Realism, criterion validity, and training capability of simulated diagnostic cerebral angiography. Studies in health technology and informatics 2014; 196: 297-303 